Is it time to approach spontaneous pneumothorax more conservatively?

\author{
A recent study provides modest evidence for \\ observational management over interventional \\ treatment, sparing patients from invasive procedures.
}

\section{PRACTICE CHANGER}

Consider observation rather than chest tube placement for primary, uncomplicated, unilateral moderate-to-large spontaneous pneumothorax in patients ages 14 to 50 .

STRENGTH OF RECOMMENDATION

B: Based on a single, lower-quality randomized controlled trial ${ }^{1}$

Brown SGA, Ball EL, Perrin K, et al; PSP Investigators. Conservative versus interventional treatment for spontaneous pneumothorax. NEngl J Med. 2020;382:405-415. doi: 10.1056/NEJMoa1910775

\section{ILLUSTRATIVE CASE}

A 26-year-old man presents to the emergency department complaining of sudden-onset left-side chest pain and mild dyspnea that started while he was playing basketball. He denies any medical problems and takes no medications. He is able to speak in complete sentences as he answers your questions. $\mathrm{His} \mathrm{O}_{2}$ saturation is $95 \%$ and a chest $x$-ray reveals a left-side, moderate-to-large pneumothorax.

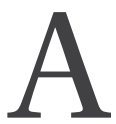
primary spontaneous pneumothorax is one that occurs in the absence of underlying clinical lung disease and is not associated with an inciting cause, such as a rib fracture. ${ }^{2}$ In the United States, the estimated incidence of primary spontaneous pneumothorax is 7.4 cases per 100,000 men and 1.2 cases per 100,000 women. ${ }^{3}$ The etiology is often unknown, but it is associated with several risk factors, includ- ing male sex, smoking, and a tall, thin body habitus. $^{2}$

The management strategy for stable patients with a primary spontaneous pneumothorax largely depends on pneumothorax size and institutional practice. Multiple methods define pneumothorax size; the US standard cutoff for a small or large pneumothorax is $3 \mathrm{~cm}$, between the pleural line and chest wall at the level of the apex, ${ }^{4}$ compared with $2 \mathrm{~cm}$ in Europe, when evaluating the distance at the hilum in an upright chest radiograph. ${ }^{5}$ The Collins method uses a formula to calculate the percentage of lung area affected based on 3 distinct measurements on a posterior/ anterior upright chest radiograph. ${ }^{6}$

Management options include observation, supplemental oxygen, simple aspiration, and thoracostomy or chest tube placement. British Thoracic Society guidelines published in 2010 state that only a small pneumothorax can be managed conservatively with observation alone; for a large pneumothorax, the guidelines recommend needle aspiration to achieve lung reinflation, followed by chest tube placement if unsuccessful. ${ }^{5}$

In practice, management of a large primary spontaneous pneumothorax varies, but the most common treatment is chest tube placement. $^{7}$ This procedure can be painful and may result in complications such as bleeding, infection, injury to internal structures, or the need for surgical intervention. ${ }^{7}$ In addi-
Gregory Jones, MD; Jeremias Georgiadis, MD; Valerie Staples, DO South Baldwin Regional Medical Center, Foley, AL

\section{Rebecca Mullen} MD, MPH

University of Colorado Family Medicine Residency, Denver

DEPUTY EDITOR

Anne Mounsey, MD Department of Family Medicine, University of North Carolina, Chapel Hill doi: $10.12788 /$ ffp. 0335 


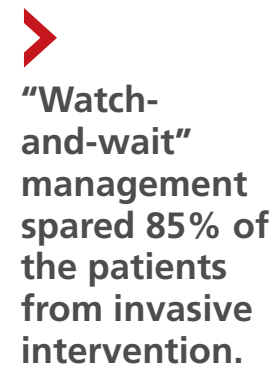

tion, once a chest tube is placed, hospital admission ensues, lasting an average of 4 days. ${ }^{8}$ Given these consequences, there is a need for safe and feasible treatment options for a large primary spontaneous pneumothorax.

\section{STUDY SUMMARY}

\section{Observational management judged noninferior, with multiple advantages}

The Primary Spontaneous Pneumothorax (PSP) trial was a prospective noninferiority trial conducted at 39 hospitals in Australia and New Zealand. This randomized controlled trial compared observational ("watch and wait") vs interventional (chest tube placement) management of uncomplicated, unilateral, primary spontaneous pneumothorax. Patients ages 14 to 50 years with a moderate-to-large pneumothorax $-32 \%$ or greater, as defined by the Collins method ${ }^{4}$ were randomly assigned to a study group to examine the primary outcome of lung reexpansion at 8 weeks.

The intervention included chest tube insertion attached to an underwater seal without suction for 1 hour, followed by an x-ray and clamping for 4 hours if there was no air leak, followed by a repeat chest x-ray. If there was no evidence of radiographic resolution, or if during observation the pneumothorax recurred, the underwater seal was recommenced and the patient was admitted to the hospital, with further intervention at the discretion of the inpatient clinicians. If radiographic improvement was seen, the tube was removed and the patient discharged.

In contrast, conservative management entailed patient observation for at least 4 hours followed by a repeat chest x-ray. If after the observation period, patients were walking comfortably and without supplemental oxygen, they were discharged. Patients in the observation group underwent an intervention if they met a variety of criteria, including unstable vitals or an enlarging pneumothorax. All patients received standard care with analgesia and supplemental oxygen as needed.

A total of 316 patients were randomized, with 154 assigned to the intervention group and 162 to the observation group. The mean age for all participants was 26. Most patients were male $(84.4 \%$ in the intervention group and $87.7 \%$ in the observation group) and almost half were current smokers $(49.3 \%$ in the intervention and $42.5 \%$ in the observation group). The mean body mass index of participants was 21.4 in the intervention and 21.3 in the observation group. Twenty-five patients (15\%) in the observation group underwent interventions for reasons specified in the research protocol (eg, "significant symptoms" such as abnormal physiologic observations and intolerable symptoms, or patient unwillingness to continue in the assigned group), and 10 patients assigned to the intervention group declined treatment.

Using a complete-case analysis, 129 of 131 patients $(98.5 \%)$ in the intervention group and 118 of 125 patients (94.4\%) in the observation group met the primary outcome of radiographic resolution within 8 weeks (risk difference $[\mathrm{RD}]=-4.1 \%$; 95\% CI, -8.6 to $0.5)$, thereby falling within the prespecified margin for noninferiority of less than $9 \%$.

Per-protocol analysis at 8 weeks also proved observational management noninferior, with 124 of 126 patients (98.4\%) in the intervention group and 123 of 130 patients $(94.6 \%)$ in the observation group achieving lung reexpansion within 8 weeks $(\mathrm{RD}=-3.8 \% ; 95 \% \mathrm{CI},-8.3$ to 0.7$)$. The time to symptom resolution was similar between groups, with a median time of 15.5 days in the intervention group compared with 14 days in the observation group (hazard ratio $=1.11$; 95\% CI, 0.88-1.4). A lower risk of serious adverse events (relative risk $[\mathrm{RR}]=3.3 ; 95 \% \mathrm{CI}$, 1.37-8.1) and pneumothorax recurrence (absolute RD $=8 \%$; 95\% CI, 0.5-15.4) occurred in the observation group vs the intervention group. The average length of hospital stay for patients in the intervention group was 6.1 days, vs 1.6 days in the observation group ( $\mathrm{RR}=2.8 ; 95 \% \mathrm{CI}, 1.8-3.6)$.

Two additional sensitivity analyses were performed because multiple study participants were lost to follow-up or had data collected after 8 weeks. Noninferiority was maintained when data collected after the 8-week visit were included and extended to 63 days ( $\mathrm{RD}=-3.7 \%$ : $95 \% \mathrm{CI},-7.9$ to 0.6 ). However, noninferiority was lost when miss- 
ing data after 8 weeks were deemed "treatment failure" (RD = $-11 \%$; 95\% CI, -18.4 to -3.5$)$.

\section{WHAT'S NEW}

\section{Conservative management enabled most patients to avoid invasive Tx risks}

In this specific patient population, conservative management of primary spontaneous pneumothorax was noninferior to interventional management and had a lower risk of serious adverse events. This management practice spared $85 \%$ of the patients from invasive intervention. As a result, they experienced a shortened hospital stay, fewer days missed from school or work, less exposure to radiation from repeat chest x-rays, and a lower rate of adverse events. Additionally, fewer of these patients had early pneumothorax recurrence.

\section{CAVEATS}

\section{There were limitations in the trial's original statistical design}

This study had a specific follow-up timetable, and some of the participants were not examined until after the 8-week checkpoint or were lost to follow-up entirely. The authors attempted to address these limitations (and show transparency) by providing additional sensitivity analyses as well as providing the intention-to-treat and per-protocol analyses for the primary outcome at 8 weeks. Noninferiority was maintained in all analyses except for the sensitivity analysis that treated missing data as treatment failure. Therefore, the authors note these approaches result in "statistical fragility" and are exploratory.

\section{CHALLENGES TO IMPLEMENTATION}

\section{Pneumothorax is not commonly} seen in outpatient settings

Family physicians working in outpatient settings generally do not encounter pneumo- thorax and, using current guidelines, would refer for emergency or inpatient care. This study opens the possibility of managing selected patients in an outpatient setting; however, this would require at least a 4-hour period of observation, which may be impractical for many outpatient-based physicians. Additionally, the study uses the Collins method to define moderate-to-large pneumothorax, which is likely an uncommon practice and thus not applicable in most primary care settings. JFP

\section{ACKNOWLEDGEMENT}

The PURLs Surveillance System was supported in part by Grant Number UL1RR024999 from the National Center for Research Resources, a Clinical Translational Science Award to the University of Chicago. The content is solely the responsibility of the authors and does not necessarily represent the official views of the National Center for Research Resources or the National Institutes of Health.

Copyright $\odot$ 2022. The Family Physicians Inquiries Network. All rights reserved.

\section{References}

1. Brown SGA, Ball EL, Perrin K, et al; PSP Investigators. Conservative versus interventional treatment for spontaneous pneumothorax. N Engl J Med. 2020;382:405-415. doi: 10.1056/ NEJMoa1910775

2. Hallifax RJ, Goldacre R, Landray MJ, et al. Trends in the incidence and recurrence of inpatient-treated spontaneous pneumothorax, 1968-2016. JAMA. 2018;320:1471-1480. doi: 10.1001/ jama.2018.14299

3. Melton LJ III, Hepper NGG, Offord KP. Incidence of spontaneous pneumothorax in Olmstead County, Minnesota: 1950 to 1974. Am Rev Respir Dis. 1979;120:1379-1382. doi: 10.1164/ arrd.1979.120.6.1379

4. Baumann MH, Strange C, Heffner JE, et al; AACP Pneumothorax Consensus Group. Management of spontaneous pneumothorax: an American College of Chest Physicians Delphi consensus statement. Chest. 2001;119:590-602. doi: 10.1378/ chest.119.2.590

5. MacDuff A, Arnold A, Harvey J; BTS Pleural Disease Guideline Group. Management of spontaneous pneumothorax: British Thoracic Society Pleural Disease Guideline 2010. Thorax. 2010;65(suppl):ii18-ii31. doi: 10.1136/thx.2010.136986

6. Collins CD, Lopez A, Mathie A, et al. Quantification of pneumothorax size on chest radiographs using interpleural distances: regression analysis based on volume measurements from helical CT. Am J Roentgenol. 1995;165:1127-1130. doi: 10.2214/ ajr.165.5.7572489

7. Kwiatt M, Tarbox A, Seamon MJ, et al. Thoracostomy tubes: a comprehensive review of complications and related topics. Int J Crit Illn Inj Sci. 2014;4:143-155. doi: 10.4103/2229-5151.134182

8. Maskell NA, Medford A, Gleeson FV. Seldinger chest drain insertion: simpler but not necessarily safer. Thorax. 2010;65:5-6. doi: 10.1136/thx.2009.117200
This study opens the possibility of managing selected patients with spontaneous pneumothorax in an outpatient setting. 